\title{
Density dependence of the momentum distributions in liquid para-hydrogen
}

\author{
J. Dawidowski, ${ }^{1, *}$ F. J. Bermejo, ${ }^{2, \dagger}$ M. L. Ristig, ${ }^{3}$ C. Cabrillo, ${ }^{4}$ and S. M. Bennington ${ }^{5}$ \\ ${ }^{1}$ Consejo Nacional de Investigaciones Científicas y Técnicas, Centro Atómico Bariloche and Instituto Balseiro, Comisión Nacional de \\ Energía Atómica, Universidad Nacional de Cuyo, (8400) Bariloche, Argentina \\ ${ }^{2}$ C.S.I.C., Department of Electricity and Electronics, University of the Basque Country, P.O. Box 644, E-48080 Bilbao, Spain \\ ${ }^{3}$ Universität zu Köln, Institut für Theoretische Physik, 50937 Köln, Germany \\ ${ }^{4}$ Instituto de Estructura de la Materia, Consejo Superior de Investigaciones Cientificas, Serrano 123 E-28006 Madrid, Spain \\ ${ }^{5}$ Rutherford Appleton Laboratory, Chilton, Didcot, Oxon, OX11 OQX, United Kingdom
}

(Received 26 January 2006; revised manuscript received 6 March 2006; published 21 April 2006)

\begin{abstract}
Momentum distributions of liquid para-hydrogen were determined by means of inelastic neutron scattering under applied pressures ranging from 1 to $80 \mathrm{bars}$, at $T=16.5 \mathrm{~K}$. The data processing procedure involves the parametrization of the dynamic structure factor and yields a set of momentum distributions as functions of the density. The results depict significant pressure dependences for all single-particle quantities such as the momentum distributions and average kinetic energies as well as for the final-state effects. The obtained results enable us to quantify the departure of the momentum distributions from classical Maxwell-Gauss shape. Such observations are then rationalized with the help of calculations that were carried out in terms of the correlated density matrix formalism.
\end{abstract}

DOI: 10.1103/PhysRevB.73.144203

PACS number(s): 67.20.+k, 61.12.-q, 61.20.Ja

\section{INTRODUCTION}

Neutron scattering measurements at large momentum transfers have proven to be the most adequate tool for the determination of single-particle properties of solids and fluids exhibiting noticeable quantum dynamics features. ${ }^{1}$ The sought quantities are the momentum distributions $n(k)$ as well as the atomic kinetic energies. The former quantities are defined as the thermal average of the number of particles having momenta with wave number $k$ at a given particle density and temperature. For condensed bodies showing strong quantum effects one expects to retrieve from experiment direct indications of quantum dynamics such as translational kinetic energies $\left\langle E_{K}\right\rangle_{T}$ well in excess of those estimated on the grounds of classical statistical mechanics-that is, $\left\langle E_{K}^{c l}\right\rangle_{T}=3 k_{B} T / 2$-as well as momentum distributions showing noticeable deviations from the Gaussian shape as predicted on the grounds of the Maxwell-Boltzmann distribution. During the last decade, such methods have provided direct quantitative information on the bounds for the Bose condensate fraction for liquid ${ }^{4} \mathrm{He}$ (Ref. 2) as well as estimates for kinetic energies calculated from the Gaussian component of $n(k)$ at $2.5 \mathrm{~K}$. The latter amounts to $15.7 \mathrm{~K}$, versus that of $\left\langle E_{K}^{c l}\right\rangle=3.75 \mathrm{~K}$, and drops to about $14 \mathrm{~K}$ upon crossing the superfluid transition.

While the case of condensed heliums is now well understood, other fluids such as the condensed hydrogens, which exhibit strong quantum features due to their low molecular masses and low temperatures characteristic of their liquid ranges, still are in need of a fully quantitative account of the importance of such quantum effects.

Previous results on liquid and solid hydrogen and deuterium ${ }^{3-7}$ have mostly dealt with the estimation of translational kinetic energies or rough estimates of the momentum distribution using large incident energies. This ensures that the incoherent limit is attained, thus minimizing the effects brought forward by the interaction of the particle once struck by the incoming neutron with the rest of the system, usually referred to as final-state effects (FSE's). In contrast, such studies are affected by the inherently low resolution achievable using incident energies of the order of a few $\mathrm{eV}$ as well as significantly excite the intramolecular vibrations, an effect that introduces some additional complications for accurately modeling of the cross sections. ${ }^{4}$ In fact, for incident neutron energies above the molecular vibrational threshold $\hbar \omega_{\nu}$ $\approx 516 \mathrm{meV}$, one expects scattering due to individual atoms rather than from the molecule as a whole and therefore two separate contributions to the kinetic energy need to be considered. The spectrum, usually transformed into a form usually referred to as a neutron Compton profile, is now a convolution of vibrational and translational components. ${ }^{4}$ On the other hand, for incident energies that are large compared to those characterizing the collective dynamics but below the vibrational threshold, ${ }^{8-10}$ the spectrum for a molecular material such as para-hydrogen which has a spherically symmetric rotational ground state ${ }^{12}$ will be comparable to that of a monatomic system which recoils with a total mass equal to the molecular mass.

The study here reported on concerns the density dependence of the single-particle properties of (mostly) liquid para-hydrogen studied at moderate-to-large momentum transfers under conditions that avoid the excitation of the first vibrational level. The present effort follows others where the spectrum of the collective excitations of both molecular hydrogen and deuterium have been described in detail ${ }^{9-11}$ with emphasis put on the characterization of the transition between collective and single-particle regimes.

The interest in the present exercise is twofold. The first concerns some recent findings pointing towards anomalies in the density dependence of some of the dynamical properties of liquid para-hydrogen. ${ }^{13}$ Second and from a more fundamental standpoint, it concerns some recent predictions made on the grounds of correlated density matrix (CDM) theory. ${ }^{14}$ 
The theory basically consists of a generalization of the correlated basis function formalism for the ground state of strongly correlated quantum fluids, ${ }^{15}$ which has been adapted to deal with nonzero temperatures. Its point of departure consists of an explicit expression for the Helmholtz free energy of liquid para-hydrogen given in terms of a functional of the radial distribution function $g(r)$ and an exchange correlation function $G_{\mathrm{cc}}(r)$ as well as the quasiparticle occupation number $n_{\mathrm{qp}}(k)$ for a given thermodynamic state. The SilveraGoldman potential ${ }^{12}$ is used to define the interparticle interactions. A variational principle then leads to the determination of the optimal $g(r), G_{c c}(r)$, and $n_{\mathrm{cc}}(k)$.

From here we may calculate the one-body reduced density matrix $^{14}(\mathrm{OBDM})$

$$
n(r)=n_{\mathrm{c}} N_{0}(r) \exp [-Q(r)]
$$

which is the dimensionless real-space Fourier transform [with $n(0)=1$ ] of the momentum distribution $n(k)$. Equation (1) facilitates a structural decomposition of $n(r)$ into a statistical exchange factor $N_{0}(r)=n_{\mathrm{cc}}(r)+N_{c}(r)$ and an exponential factor where the short-ranged function $Q(r)$ embodies the spatial correlations between the phase factors associated with the single-particle wave functions in the correlated state of the quantum fluid. The strength factor $n_{\mathrm{c}}=\exp [Q(0)]$ is closely related to the total kinetic energy of the liquid. The exchange factor $N_{0}(r)$ consists of the OBDM element $n_{\mathrm{cc}}(r)$ of quasiparticles and an additional term $N_{c}(r)$ which collects all other possible exchange correlations.

Application of the CDM algorithm in conjunction with the hypernetted-chain technique ${ }^{14}$ permits a fast and efficient enumeration of the quantities involved in Eq. (1). For liquid para-hydrogen close to the triple point the contribution $N_{c}(r)$ is very small and may be ignored. Further, the quasiparticle distribution $n_{\mathrm{cc}}(r)$ follows the classical Boltzmann form

$$
n_{\mathrm{cc}}(r)=\exp \left[-\pi\left(\frac{r}{\lambda}\right)^{2}\right]
$$

of independent and distinguishable particles with molecular hydrogen mass $M$ and thermal wavelength $\lambda$. Consequently, Eq. (1) specializes to the expression

$$
n(r)=n_{\mathrm{c}} \exp \left[-\pi\left(\frac{r}{\lambda}\right)^{2}-Q(r)\right] .
$$

The function (3) is of substantially shorter range than the quasiparticle distribution $n_{\mathrm{cc}}(r)$ and, moreover, deviates from a Gaussian form. Put into other words, the prediction (3) tells (once viewed in momentum space) that the molecular interactions drastically reduce the number of molecules having small momenta by a factor of about $1 / 3$, shifting them towards intermediate and large momenta. This effect is also reflected in the kinetic energy distribution measured by $k^{2} n(k)$ and, in particular, by the large value of the total kinetic energy per molecule,

$$
\left\langle E_{K}\right\rangle_{T}=\frac{1}{2 M} \int d k k^{2} n(k) .
$$

The energy (4) significantly exceeds the energy value of its classical analog calculated from the integral (4) where $n(k)$ is replaced by $n_{\mathrm{cc}}(k)$.

It is instructive to compare the kinetic energy distribution $k^{2} n(k)$ with the familiar classical Maxwell-Gauss distribution $k^{2} n_{\mathrm{G}}(k)$ with an associated total kinetic energy $\left\langle E_{K}^{G}\right\rangle_{T}$ $=\left\langle E_{K}\right\rangle_{T}$. The classical momentum distribution $n_{\mathrm{G}}(k)$ and its Fourier inverse $n_{\mathrm{G}}(r)$ are, of course, represented by Gaussian functions with an effective thermal wavelength $\lambda_{\mathrm{G}}$ due to the kinetic energy condition. Obviously, the difference

$$
\Delta(k)=k^{2}\left[n(k)-n_{\mathrm{G}}(k)\right]
$$

measures the deviation of the kinetic energy density of the $\mathrm{H}_{2}$ liquid from the classical Maxwell distribution. Thus, Eq. (5) provides quantitative information on the effects caused by the quantum-mechanical indistinguishability of identical particles.

Here, we set ourselves to provide an experimental measurement that permits a test of the theoretical predictions (1) and (3) of CDM theory. As mentioned above and to the authors' knowledge there are no results yet available to carry out a scrutiny of the theoretical prediction.

\section{EXPERIMENT}

The neutron measurements were performed using the MARI spectrometer at the ISIS pulsed neutron source (Rutherford Appleton Laboratory), and a temperature of $16.5 \mathrm{~K}$ was selected. A neutron incident energy of $450 \mathrm{meV}$ was chosen to explore a wide region in the momentum-energy map, while avoiding exciting vibrationally the intramolecular mode. The sample holder was an aluminum pressure cell consisting of a hollow cylinder, $37 \mathrm{~mm}$ inner diameter, $46.6 \mathrm{~mm}$ outer diameter, and $58 \mathrm{~mm}$ of height, exposed to the beam. The inner-wall thickness was $1.7 \mathrm{~mm}$ and the outer $1 \mathrm{~mm}$. The sample was obtained from high-purity hydrogen gas transformed to para $-\mathrm{H}_{2}$ by forcing it to pass through an activated catalyst, as described elsewhere. ${ }^{10}$ For the first measurement, gas was pumped to pressurize the sample at 80 bars, and subsequently, the gas was released to perform measurements at 70,60,50,40,30,15, and 1 bars, respectively. An empty-cell measurement was also performed, as well as a vanadium measurement to follow the standard MARI calibration procedure. In Fig. 1 we show the experimental data from a typical run in the $Q-\omega$ plane at 80 bars, after performing the corrections described in the next section.

\section{EXPERIMENTAL DATA PROCESSING}

\section{A. Preliminary processing}

The experimental data measured as a function of the scattering angle and the time of flight were transformed into the experimental magnitude $s(Q, \varepsilon)$ defined elsewhere, ${ }^{16}$ employing standard MARI procedures. 

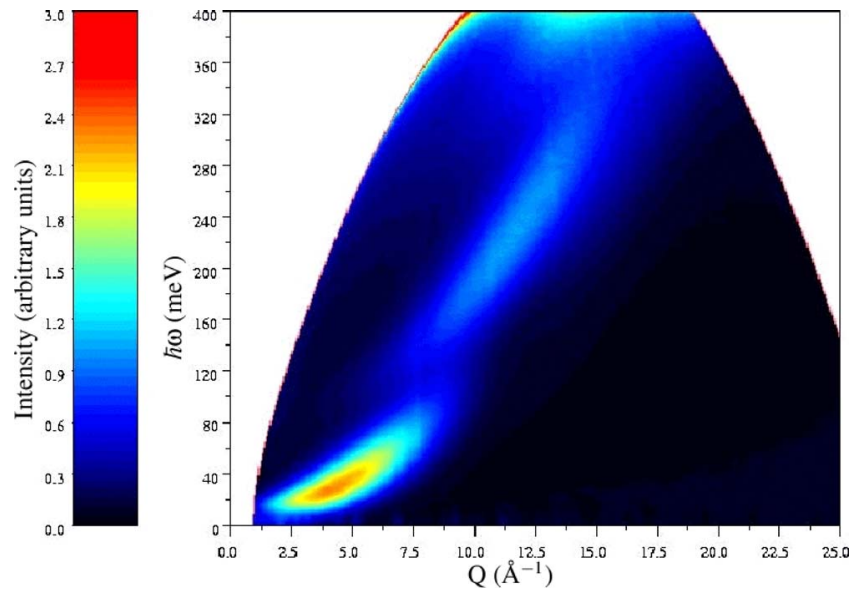

FIG. 1. (Color online) Color map showing the scattering law in the $Q-\omega$ plane of the experimental data for liquid para- $\mathrm{H}_{2}$ at $p$ $=80 \mathrm{bars}$ and $T=16.5 \mathrm{~K}$, after performing the multiple-scattering and attenuation corrections.

\section{B. Multiple-scattering and attenuation corrections}

Multiple-scattering and attenuation corrections were performed following steps described in detail in Ref. 16, so we will give only a brief outline here. The measured quantity is the double-differential macroscopic cross section-i.e., the probability that an incident neutron with a wave vector $\mathbf{k}_{0}$ will emerge from the sample-container set with a wave vector k. Its explicit form is given by

$$
\frac{d^{2} \Sigma}{d \Omega d E}=\frac{N \sigma_{b}}{4 \pi A} \frac{k}{k_{0}} s(Q, \varepsilon),
$$

where $s(Q, \varepsilon)$ is the macroscopic scattering law proposed by Sears, dependent on the geometry and the scattering properties of the sample. ${ }^{17}$ In turn, it can be decomposed into a part due to singly scattered neutrons by the sample $s_{1}(Q, \varepsilon)$, another due to single scattering from the can $s_{C}(Q, \varepsilon)$, and a third due to multiply scattered neutrons (with any combination of sample-can-scattering events) $s_{M}(Q, \varepsilon)$ :

$$
s(Q, \varepsilon)=s_{1}(Q, \varepsilon)+s_{M}(Q, \varepsilon)+s_{C}(Q, \varepsilon) .
$$

This quantity is closely related to the data contained in the experimental data set $\mathcal{E}(Q, \varepsilon)$ :

$$
s(Q, \varepsilon)=\frac{2 A k_{0}^{2}\left[1-\mathcal{T}\left(E_{0}\right)\right]}{\alpha N \sigma_{b}} \mathcal{E}(Q, \varepsilon),
$$

where $A$ is the cross-sectional area of the sample perpendicular to the incident beam, $\mathcal{T}\left(E_{0}\right)$ is the fraction of transmitted neutrons at incident energy $E_{0}, \alpha$ is a normalization constant, and $N$ is the total number of scattering centers. ${ }^{16}$

On the other hand, $s_{1}(Q, \varepsilon)$ defined in Eq. (7) is simply related to the sought scattering law $S(Q, \varepsilon)$ through

$$
s_{1}(Q, \varepsilon)=S(Q, \varepsilon) H(Q, \varepsilon),
$$

where $H(Q, \varepsilon)$ is the attenuation factor-i.e., the fraction of undetected singly scattered neutrons due either to multiple scattering or neutron absorption or due to finite detector efficiency.

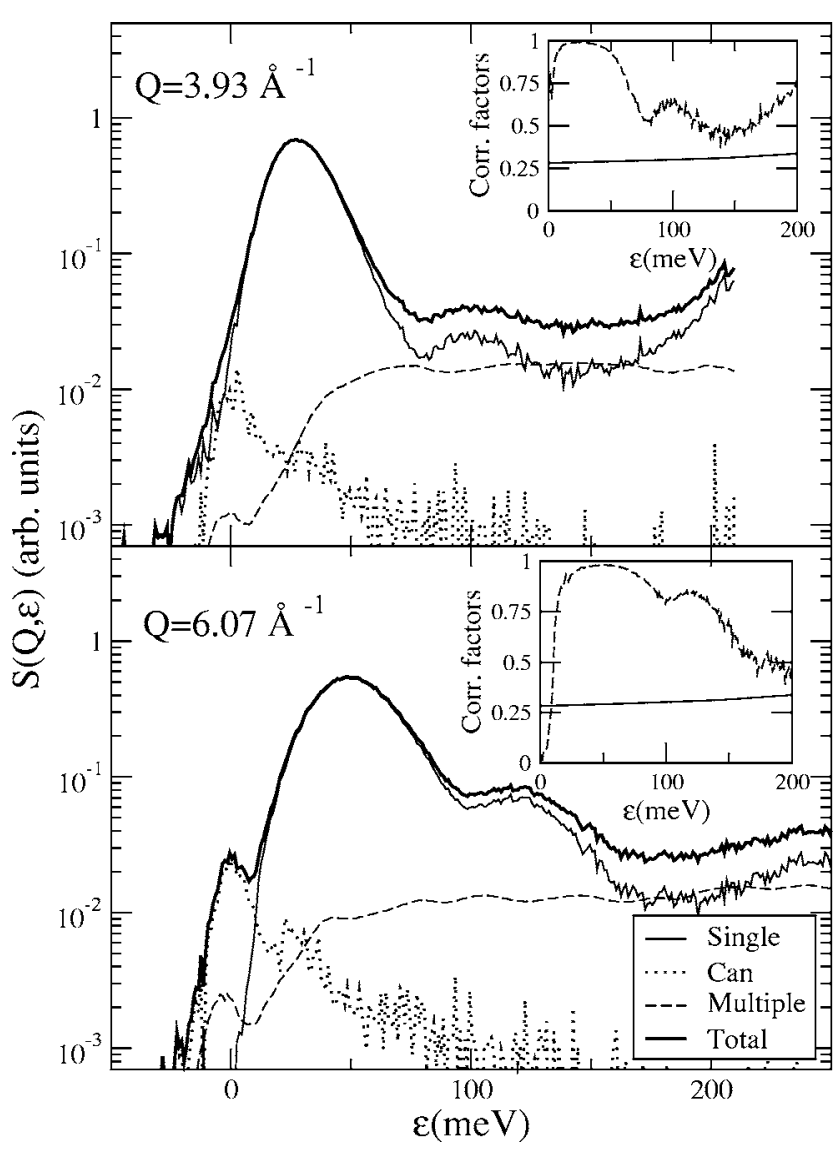

FIG. 2. Simulation results for liquid hydrogen at $p=1$ bar, for two different $Q$ values. Thin solid line, single scattering; dotted line, can contribution; dashed line, multiple scattering; thick solid line, total scattering. Insets: attenuation coefficient (solid line) and multiple-scattering coefficient (dashed line).

The correction procedure is based upon an iterative scheme that employs Monte Carlo simulations, following some of the steps proposed by Copley. ${ }^{18}$ Neutron histories are individually monitored, and at each step the free path traveled by the neutron is obtained from distributions of mean free paths of the materials traversed at the current energy $E$. Such distributions are tabulated as input and are based on experimental data. The choice of different energies and flight directions at each step is based upon the experimental raw scattering laws deduced from measurements carried out for the sample and empty-container measurements in the first run and in the corrected distributions in the subsequent runs. After a run, two correction factors are defined as a function of $Q$ and $\varepsilon$ : namely, the attenuation factor defined in Eq. (9) and a single-to-multiple scattering ratio. Such factors are applied to the raw experimental data to produce a different distribution function that is employed in the next iteration. ${ }^{16}$ The iterative process continues until no significant differences in the multiple-scattering and container contributions are observed between iterations. Typically, for the data analyzed in the present paper, four iterations were needed to reach convergence.

As an example, in Fig. 2 we show the contributions of single and multiple scattering, as well as of the neutrons scattered by the container for the case of the measurement at 


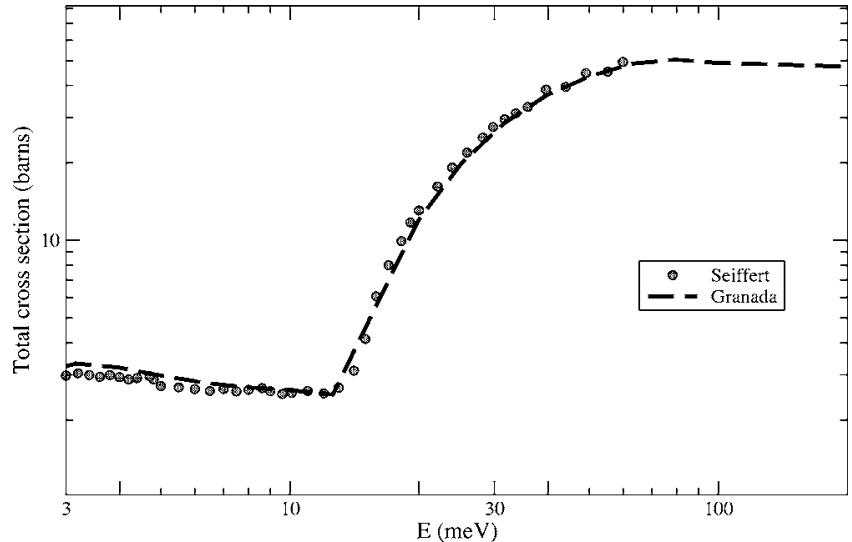

FIG. 3. Total scattering cross section of liquid hydrogen according to Seiffert's experimental data and the model of Granada for a $0.1 \%$ content of ortho-Hydrogen.

1 bar for two different values of $Q$. In the insets we show the corresponding attenuation and multiple-scattering coefficients.

\section{Determination of para- and ortho-hydrogen contents}

An approximate knowledge of the para- and orthohydrogen contents is needed for the ensuing discussions. In a previous work $^{10}$ this was determined by observing the $S(Q)$ obtained by constant $Q$ integration of the $S(Q, \omega)$. This was possible, since the low incident neutron energy $(34.8 \mathrm{meV})$ provided a sufficiently narrow resolution function to observe the first diffraction peak at $\approx 2 \AA^{-1}$. However, in the present configuration the resolution function is far wider, preventing such determination. Because of this, we have followed a different route for the estimation, based upon previous experimental knowledge for the total cross section ${ }^{19}$ and the theoretical model developed by Granada et al. ${ }^{20}$ In Fig. 3 we show Seiffert's experimental data, together with a calculation carried out on the basis of the model of Granada et al. corresponding to a $0.1 \%$ contents of ortho-hydrogen. The excellent agreement shown in the figure guarantees the adequacy of the model of Granada et al. to assess the ortho-hydrogen contents. On the other hand, the $S(Q, \omega)$ measured in the present experiment allows us to calculate the total scattering cross sections according to ${ }^{21}$

$$
\sigma\left(E_{0}\right)=\frac{\sigma_{b}}{2 k_{0}^{2}} \int_{0}^{\infty} Q \int_{\varepsilon_{\min }}^{\varepsilon_{\max }} S(Q, \varepsilon) d \varepsilon
$$

where the integration limits are $\varepsilon_{\max }=\frac{\hbar^{2} k_{0} Q}{m}\left(1-\frac{Q}{2 k_{0}}\right)$ and $\varepsilon_{\min }$ $=-\frac{\hbar^{2} k_{0} Q}{m}\left(1+\frac{Q}{2 k_{0}}\right), m$ is the neutron mass, and $\sigma_{b}$ is the boundatom hydrogen-scattering cross section.

The next step consists in estimating the ortho-para contents by calculating the total cross sections with the model of Granada et al. and comparing the result with our experimental data as shown in Fig. 4, where the estimated orthohydrogen content is shown for each measured pressure.

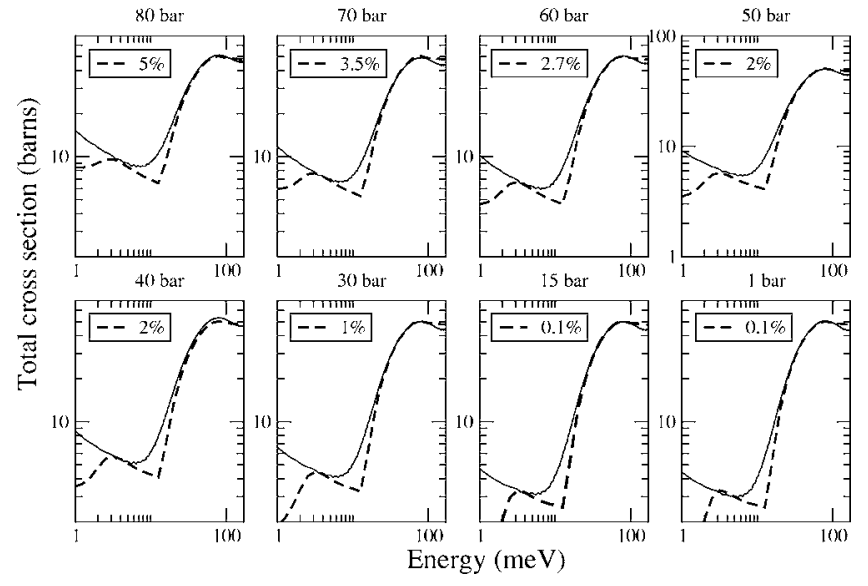

FIG. 4. Total scattering cross section of liquid hydrogen obtained at different pressures from our measurements, matched with the calculation of the model of Granada et al. with the indicated ortho- $\mathrm{H}_{2}$ contents.

\section{DATA ANALYSIS}

\section{A. Basic equations}

The data analysis route here followed to study the momentum distributions is based upon the formalism developed by Glyde and others. ${ }^{22}$ The useful range of $Q$ values upon which we base our analysis starts at $4.9 \AA^{-1}$, where the oscillations in the intermolecular (or center-of-mass) structure factor are significantly damped out. ${ }^{23}$ On such grounds, we can safely describe the center-of-mass scattering law by its self-component, described by the series expansion

$$
\begin{aligned}
S_{\text {self }}(Q, \omega)= & S_{\mathrm{IA}}(Q, \omega)\left[1+S_{1}(Q, \omega)+S_{2}(Q, \omega)\right. \\
& \left.+S_{3}(Q, \omega)+S_{4}(Q, \omega)\right],
\end{aligned}
$$

where $S_{\mathrm{IA}}(Q, \omega)$ is the scattering law in the impulse approximation,

$$
S_{\mathrm{IA}}(Q, \omega)=\frac{S(Q)}{\sqrt{2 \pi \mu_{2}}} \exp \left[-\hbar^{2}\left(\omega-\omega_{R}\right)^{2} / 2 \mu_{2}\right]
$$

$\omega_{R}=\frac{\hbar^{2} Q^{2}}{2 M_{m o l}}$, is the energy transfer referred to the recoil energy, and $M_{m o l}$ is the hydrogen molecular mass. The rest of the terms in Eq. (11) are related to the parameters $\mu_{2}, \mu_{3}, \mu_{4}, \mu_{5}$, and $\mu_{6}$ which are the first five cumulants defined and extensively studied in Ref. 22:

$$
\begin{gathered}
S_{1}(Q, \omega)=-\frac{\mu_{3}}{2 \mu_{2}^{2} z}\left[1-\frac{z^{2}}{2 \mu_{2}}\right], \\
S_{2}(Q, \omega)=\frac{\mu_{4}}{8 \mu_{2}^{2}}\left[1-2 \frac{z^{2}}{\mu_{2}}+\frac{z^{4}}{3 \mu_{2}^{2}}\right], \\
S_{3}(Q, \omega)=\frac{\mu_{5}}{8 \mu_{2}^{3} z}\left[1-\frac{2 z^{2}}{3 \mu_{2}}+\frac{z^{4}}{15 \mu_{2}^{2}}\right],
\end{gathered}
$$




$$
S_{4}(Q, \omega)=-\frac{5}{24 \mu_{2}^{3}}\left(\frac{\mu_{6}}{10}+\mu_{3}^{2}\right)\left[1-\frac{3 z^{2}}{\mu_{2}}+\frac{z^{4}}{\mu_{2}^{2}}-\frac{z^{6}}{15 \mu_{2}^{3}}\right],
$$

where $z=\hbar\left(\omega-\omega_{R}\right)$. We will briefly refer to the physical meaning of the cumulants $\mu_{2}, \mu_{3}$, and $\mu_{4}$, which will be relevant in further analysis. The parameter $\mu_{2}$ is related to the width $\bar{\alpha}_{2}$ of the Gaussian that results as a first approximation of $S(Q, \omega)$ within the impulse approximation, which is the mean value of the momentum of the struck atom in the direction of $\mathbf{Q}$-i.e., $\left\langle k_{Q}^{2}\right\rangle$,

$$
\mu_{2}=\hbar^{2} q^{2} \bar{\alpha}_{2}=q^{2}\left\langle k_{Q}^{2}\right\rangle,
$$

where $q=\hbar Q / M$ and $M$ is the molecular mass. $\mu_{3}$ is defined as

$$
\mu_{3}=\hbar^{3} q^{2} \bar{a}_{3}=q^{2}\left\langle\nabla^{2} \nu(r)\right\rangle / 6 \hbar,
$$

where $\nu(r)$ is the interparticle potential on the struck atom, and

$$
\mu_{4}=\hbar^{4} q^{2} \bar{a}_{4}+\hbar^{4} q^{4} \bar{\alpha}_{4},
$$

where $\bar{\alpha}_{4}=\left[\left\langle k_{Q}^{4}\right\rangle-3\left\langle k_{Q}^{2}\right\rangle^{2}\right]$ and $\bar{a}_{4}=\left\langle F_{Q}^{2}\right\rangle / 3 \hbar^{2}, F_{Q}$ being the force on the struck atom along the scattering vector $\mathbf{Q}$. It should be noticed that while $\bar{\alpha}_{4}$ can be positive or negative, $\bar{a}_{4}$ is always positive. ${ }^{22}$

Thus, the complete scattering law for liquid hydrogen can be described by the formalism extensively developed in the literature ${ }^{24,25}$ which we will briefly describe. Let us consider a sample consisting of a mixture with a concentration $c$ of ortho- and $(1-c)$ of para-hydrogen. We will examine the self-contribution (i.e., the contributions of the neutrons scattered from the same molecular center of mass). Let $a_{c}$ and $a_{i}$ be, respectively, the coherent and incoherent scattering lengths of hydrogen, $r_{e}$ the equilibrium distance between hydrogen atoms in the molecule, $B$ the rotational constant, and the functions $j_{\ell}(x)$ the spherical Bessel functions of $\ell$ th order; then, the para-contribution of the first significant terms in the rotational modes expansion is

$$
T_{\text {para-1 }}(Q, \omega)=4(1-c) a_{i}^{2}\left[3 j_{1}^{2}\left(Q r_{e} / 2\right) S_{\text {self }}(Q, \omega-2 B)\right],
$$

$$
T_{\text {para- } 2}(Q, \omega)=4(1-c) a_{i}^{2}\left[5 j_{2}^{2}\left(Q r_{e} / 2\right) S_{\text {self }}(Q, \omega-6 B)\right],
$$

$$
T_{\text {para-3 }}(Q, \omega)=4(1-c) a_{i}^{2}\left[7 j_{3}^{2}\left(Q r_{e} / 2\right) S_{\text {self }}(Q, \omega-12 B)\right],
$$

$$
T_{\text {para- } 4}(Q, \omega)=4(1-c) a_{i}^{2}\left[9 j_{4}^{2}\left(Q r_{e} / 2\right) S_{\text {self }}(Q, \omega-20 B)\right],
$$

$$
T_{\text {para-5 }}(Q, \omega)=4(1-c) a_{i}^{2}\left[11 j_{5}^{2}\left(Q r_{e} / 2\right) S_{\text {self }}(Q, \omega-30 B)\right],
$$

where the subscript "para- $\ell$ " means that the initial state of the molecule is para-hydrogen, and it acquires an angular
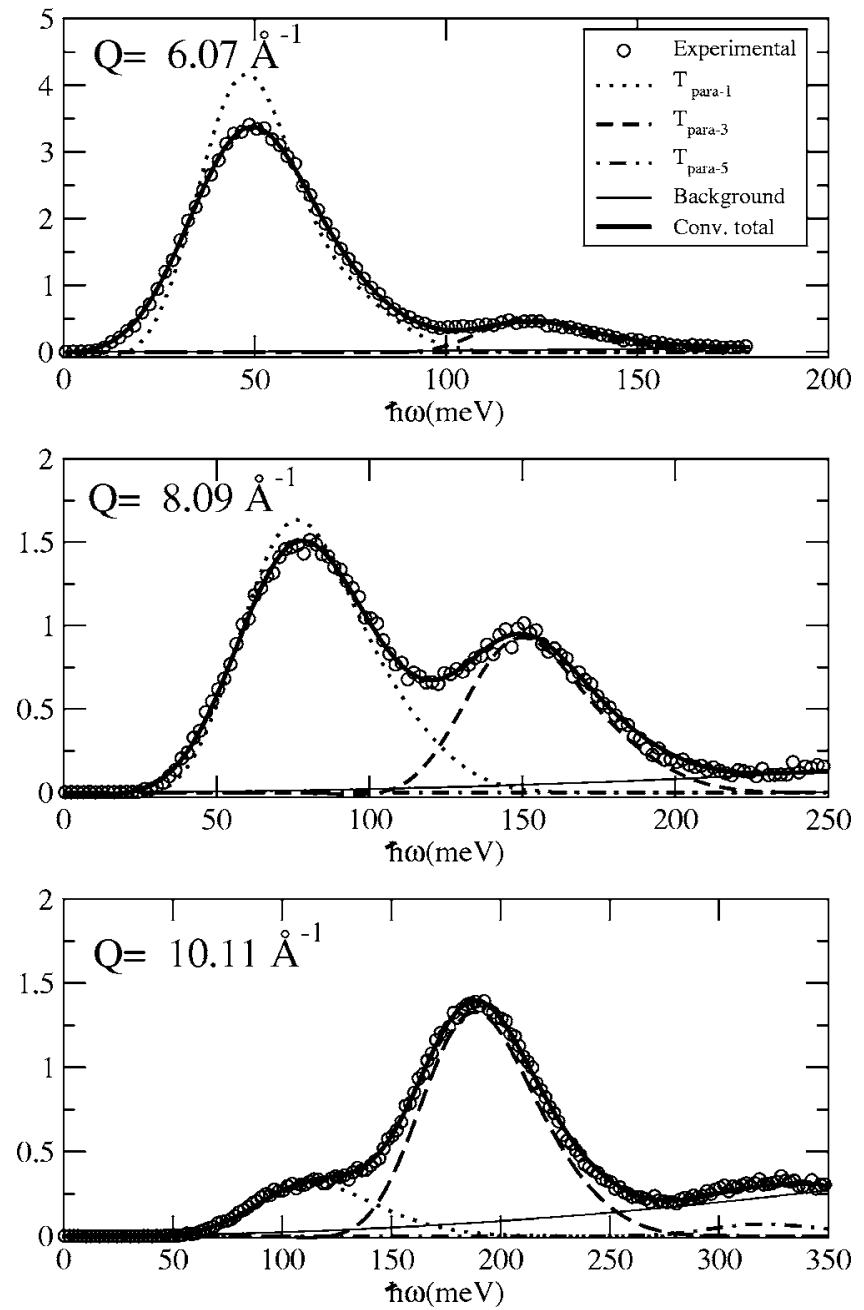

FIG. 5. Typical results of the fitted curves for liquid hydrogen at 1 bar. Only the significant terms of Eq. (27) are plotted. The total fitted curve is convoluted with the resolution function. The contribution of $T_{\text {para-3 }}(Q, \omega)$ is noticeable only in the lower frame.

momentum $\ell$ in its final state. It should be noted that Eqs. (20)-(24) do not include molecular vibrational excitations as commented above.

The contribution of ortho-hydrogen can be decomposed in the same way into a series of terms of the rotational mode. The first significant one of those becomes

$$
\begin{aligned}
T_{\text {ortho }}(Q, \omega)= & 4 c\left\{( a _ { c } ^ { 2 } + \frac { 2 } { 3 } a _ { i } ^ { 2 } ) \left\{2 j_{2}^{2}\left(Q r_{e} / 2\right) S_{\text {self }}(Q, \omega)\right.\right. \\
& \left.+\left[3 j_{2}^{2}\left(Q r_{e} / 2\right)+4 j_{4}^{2}\left(Q r_{e} / 2\right)\right] S_{\text {self }}(Q, \omega-10 B)\right\} \\
& +\frac{a_{i}^{2}}{3}\left\{2 j_{0}^{2}\left(Q r_{e} / 2\right) S_{\text {self }}(Q, \omega)\right. \\
& +j_{1}^{2}\left(Q r_{e} / 2\right) S_{\text {self }}(Q, \omega+2 B) \\
& \left.\left.+\left[2 j_{1}^{2}\left(Q r_{e} / 2\right)+3 j_{3}^{2}\left(Q r_{e} / 2\right)\right] S_{\text {self }}(Q, \omega-4 B)\right\}\right\} .
\end{aligned}
$$




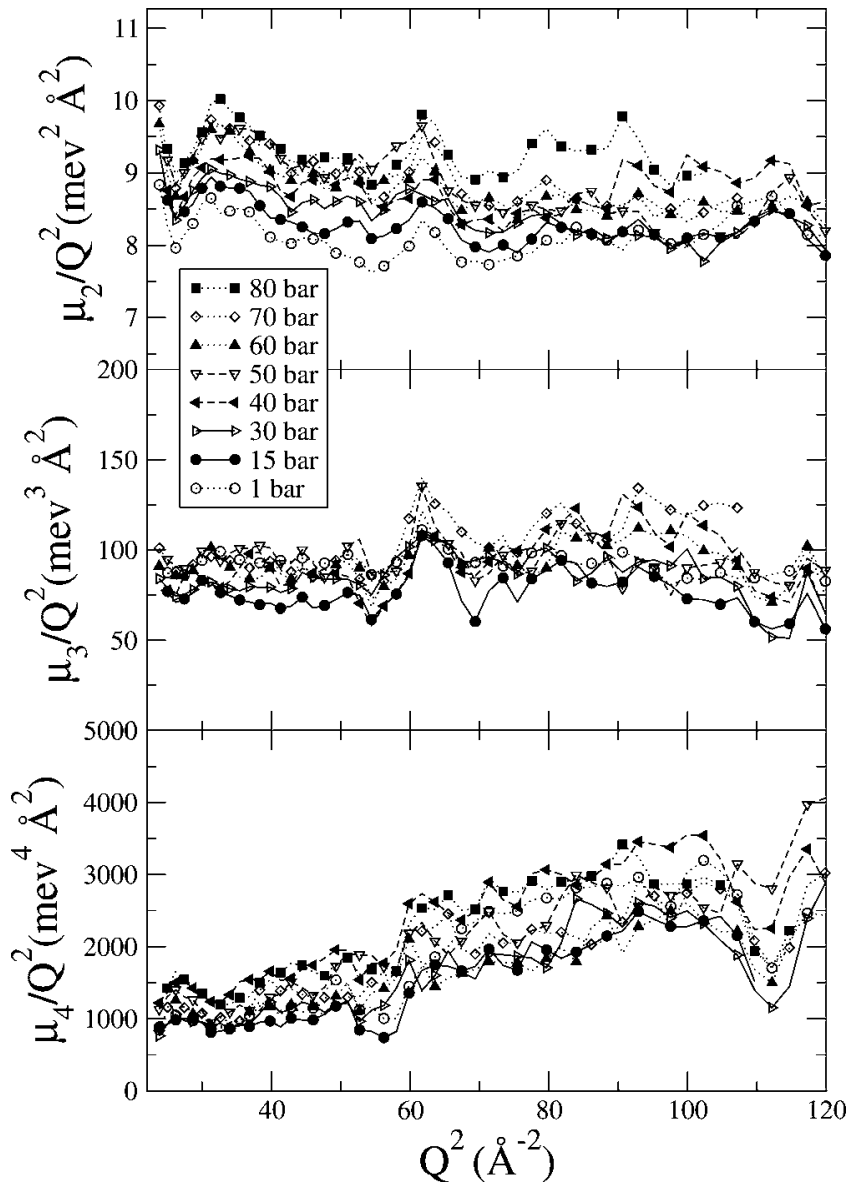

FIG. 6. Parameters $\mu_{2} / Q^{2}, \mu_{3} / Q^{2}$, and $\mu_{4} / Q^{2}$ fitted at different pressures.

Finally, the coherent contribution (i.e., the contribution of the interference of neutrons scattered from different molecular centers) is

$$
T_{\text {coh }}(Q, \omega)=4 a_{c}^{2} 3 j_{0}^{2}\left(Q r_{e} / 2\right) S_{\text {self }}(Q, \omega) .
$$

The complete scattering law to fit to our experimental data results from collecting Eqs. (25) and (26):

$$
\begin{aligned}
T(Q, \omega)= & A\left[T_{\text {para-1 }}(Q, \omega)+T_{\text {para-2 }}(Q, \omega)+T_{\text {para-3 }}(Q, \omega)\right. \\
& +T_{\text {para-4 }}(Q, \omega)+T_{\text {para-5 }}(Q, \omega)+T_{\text {coh }}(Q, \omega) \\
& \left.+T_{\text {orto }}(Q, \omega)\right],
\end{aligned}
$$

where $A$ is a scale factor and each one of the terms in Eq. (27) contains the above-described parameters. In total four parameters were fitted.

\section{B. Data fitting}

Experimental data corrected as described in Secs. III A and III B were fitted to Eq. (27) convoluted with the measured MARI resolution function for the present configuration. In Fig. 5 we show examples of such fittings, with the contribution of the most significant terms of Eq. (27). Attempts to employ Eq. (11) up to the term $S_{4}(Q, \omega)$ resulted in a slowly converging process, so we decided to consider up to
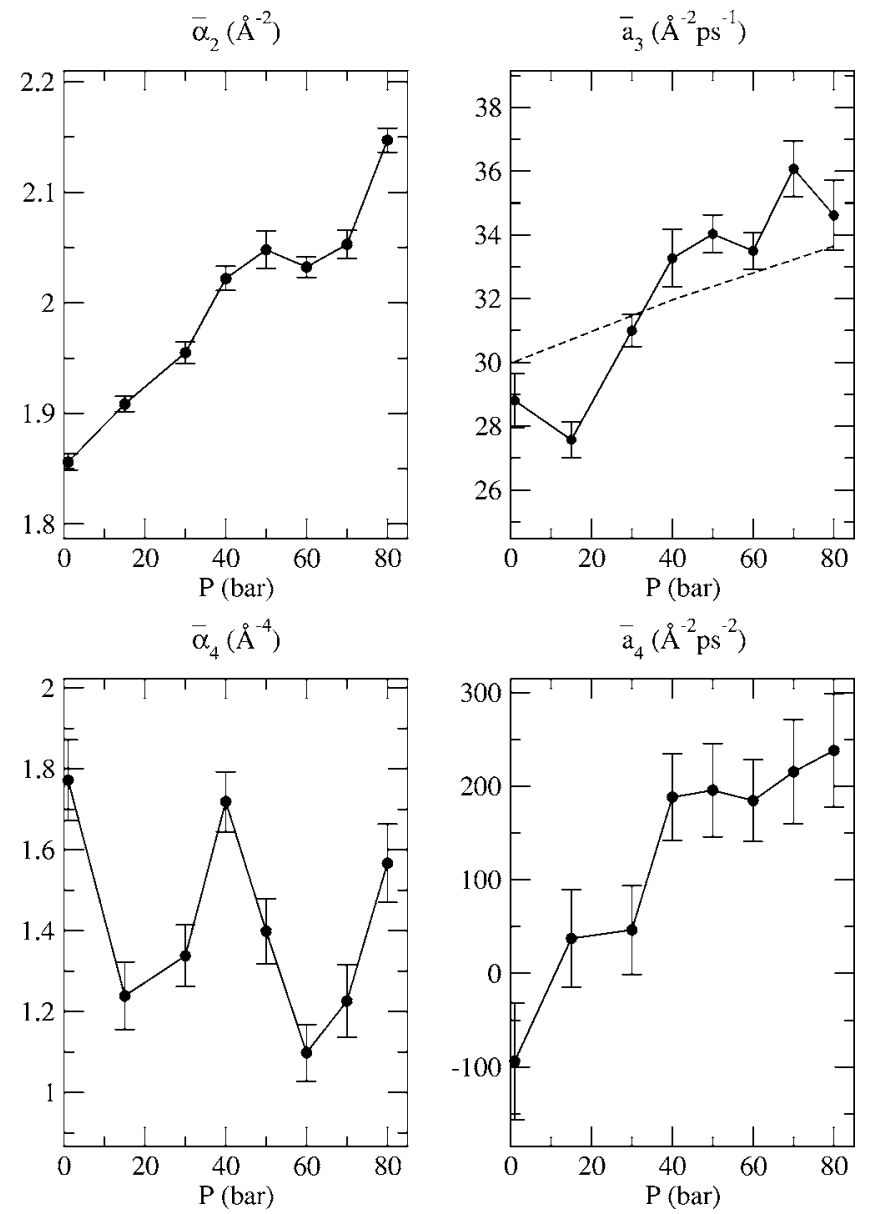

FIG. 7. The fitted parameters $\bar{\alpha}_{2}, \bar{a}_{3}, \bar{a}_{4}$, and $\bar{\alpha}_{4}$. The parameter $\bar{a}_{3}$ is compared with a calculation based on the intermolecular potential (see text for details).

the $S_{3}(Q, \omega)$ term only. This choice was useful, since the dynamical features we want to extract from the data are essentially contained within the parameters $\mu_{2}, \mu_{3}$, and $\mu_{4}$, which are included in the terms $S_{1}(Q, \omega)$ and $S_{2}(Q, \omega)$. On the other hand, the fitting process showed a coupling between terms of equal parity, so $S_{1}$ was affected by $S_{3}$.

A first round of data fittings allowed us to define the parameters $\mu_{2}$ and $\mu_{3}$ as shown in Fig. 6. The parameters are plotted as divided by $Q^{2}$ following the procedure described by Glyde $^{22}$ to detect any residual wave-vector dependence.

A glance to Fig. 6 shows that rather than exhibiting welldefined oscillations in $\mu_{2,3}(Q) / Q^{2}$ indicative of the presence of coherent effects, the curves show smooth trends, or in other words, the obtained values are basically independent of the momentum transfer. A linear increase is observed for $\mu_{4}(Q) / Q^{2}$ as a function of $Q^{2}$ in Fig. 6, and this allows a description based on Eqs. (17)-(19) as discussed by Glyde. ${ }^{22}$ We thus have attempted to fit all data using the functional forms of Eqs. (17)-(19). To do so and thus to reduce the number of adjustable parameters, an iterative fitting method was employed. In the first step, the preliminary values obtained for $\mu_{2}$ are introduced in Eq. (27) [employing Eq. (17)], thus fitting only $\mu_{3}$ and $\mu_{4}$. The values of $\mu_{4}$ are again fitted with Eq. (19), and the functional form (19) is intro- 


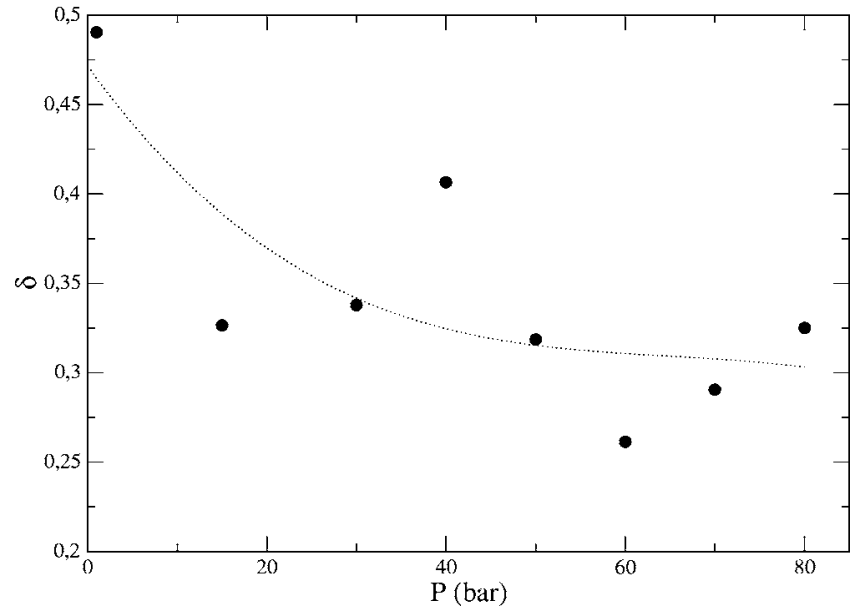

FIG. 8. Kurtosis of the observed peaks in $S(Q, \omega)$ as a function of the pressure. The dotted line is a guide for the eye.

duced again in (27) to perform a fit of $\mu_{2}$. After such process we obtained the results of $\bar{\alpha}_{2}, \bar{a}_{3}, \bar{a}_{4}$, and $\bar{\alpha}_{4}$ shown in Fig. 7 .

From the obtained values for $\bar{a}_{3}$ and $\bar{a}_{4}$ an estimate of the strength of final-state effects may be derived. These are often cast in terms of a final-state broadening function $R(Q, \omega)$ which measures the departure of the observed spectrum from that satisfying the impulse approximation $S_{\mathrm{IA}}(Q, \omega)$,

$$
S(Q, \omega)=S_{\mathrm{IA}}(Q, \omega) \otimes R(Q, \omega),
$$

so that if the impulse approximation were to hold exactly, $R(Q, \omega)=\delta(\omega)$. From Ref. 22, the first two nonzero moments of $R(Q, \omega)$ are exactly given by $\mu_{3}=q^{2} \bar{a}_{3}$ and $\mu_{4}-\alpha_{4}=q^{2} \bar{a}_{4}$. We cannot perform the Fourier transform required to calculate $R(Q, \omega)$ from knowledge of those momenta, since $\mu_{4}$ $-\alpha_{4}$ is positive, so higher momenta would be needed, and as stated above, they cannot be easily extracted from the data.

\section{Momentum distributions}

Following Glyde, ${ }^{22}$ the momentum distributions can be calculated from the parameters $\bar{\alpha}_{2}$ and $\bar{\alpha}_{4}$ :

$$
n(k)=\frac{e^{-k^{2} / 2 \bar{\alpha}_{2}}}{\left(2 \pi \bar{\alpha}_{2}\right)^{3 / 2}}\left[1+\frac{\delta}{8}\left(5-10 \frac{k^{2}}{3 \bar{\alpha}_{2}}+\frac{k^{4}}{3 \bar{\alpha}_{2}^{2}}\right)\right],
$$

where

$$
\delta=\frac{\bar{\alpha}_{4}}{\bar{\alpha}_{2}^{2}}
$$

is the kurtosis of the distribution. In Fig. 8 we show its values as a function of the pressure.

Equation (29) represents a Gaussian distribution corrected by the factor in square brackets, which represents the nonGaussian contribution. The momentum distributions obtained in this work are nearly Gaussian. In Fig. 9 we represent the Gaussian contribution to the momentum distributions (upper frame), the non-Gaussian corrections (middle frame), and the total distributions (lower frame).

The mean kinetic energy can readily be obtained from ${ }^{3}$

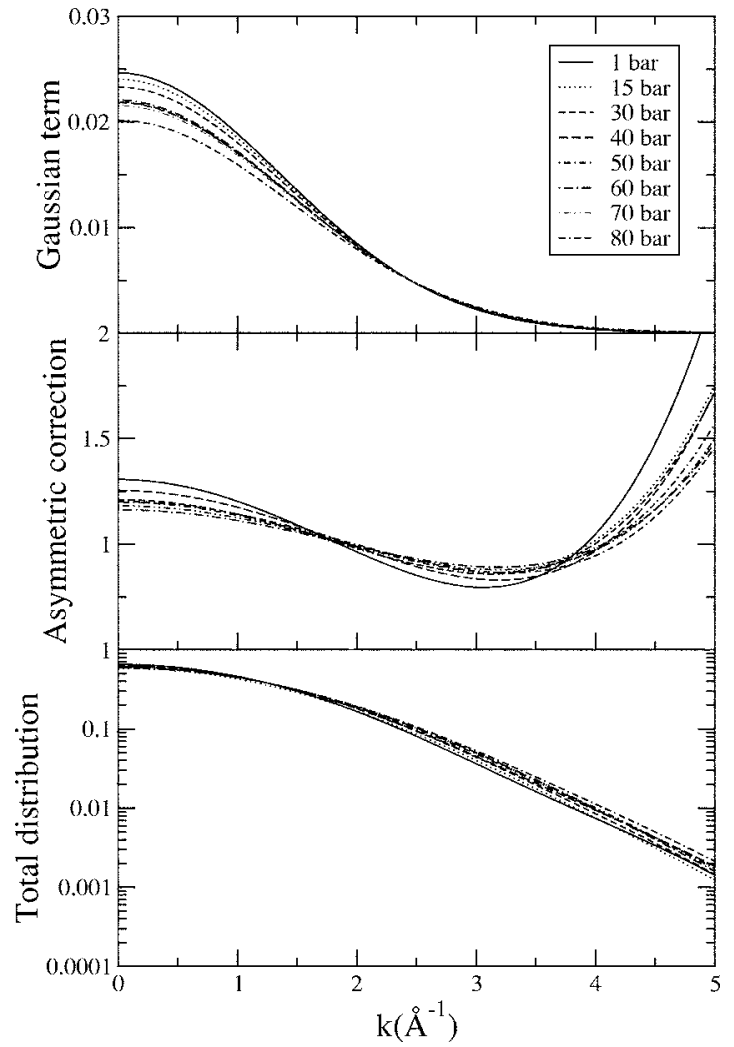

FIG. 9. Upper frame: the Gaussian contribution to the momentum distributions. Middle frame: the non-Gaussian corrections represented by the square bracket of Eq. (29). Lower frame: the total distributions.

$$
\left\langle E_{K}\right\rangle=\frac{3}{2} \frac{\hbar^{2}}{M} \bar{\alpha}_{2}
$$

In Fig. 10 we show the kinetic energy as a function of the pressure. The values range from $67.8 \mathrm{~K}$ at room pressure to $77.5 \mathrm{~K}$ at 80 bars, to be compared with the value $(63 \pm 6) \mathrm{K}$ reported in Ref. 3 at $17 \mathrm{~K}$.

\section{DISCUSSION}

\section{A. Density dependence of the kinetic energy}

Here we comment on the observed increase of about $19 \%$ in particle kinetic energy with increasing density. The latter is known to raise a mere $8 \%$ within the range of applied pressures here employed. On general grounds that are valid for either quantum or classical liquids one expects the kinetic energy to follow

$$
\left\langle K_{E}\right\rangle_{T}=\sum_{j}\left(\frac{\hbar^{2} Q^{2}}{2 M}\right) n_{j} / \sum_{j} n_{j},
$$

where $n_{j}$ stands for the Bose occupation function,

$$
n_{j}=\frac{1}{\exp \beta\left(\varepsilon_{j}-\mu\right)-1},
$$

with $\varepsilon_{j}=\hbar^{2} Q^{2} / 2 M, \mu$ is the chemical potential, and $\beta$ $=1 / k_{B} T$. For a classical liquid where Boltzmann statistics 


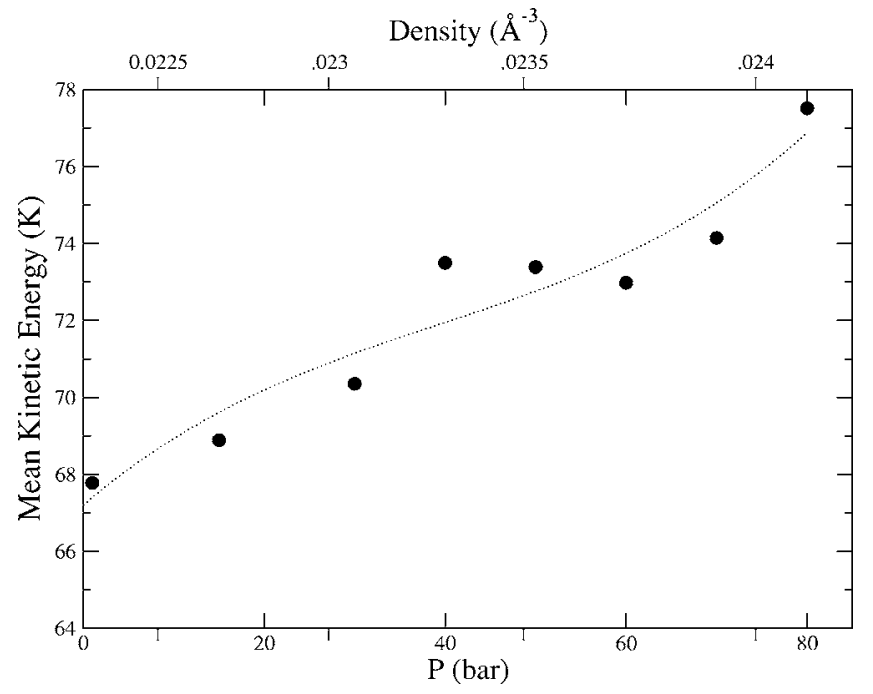

FIG. 10. Kinetic energy as a function of pressure (lower abscissa) or density (upper abscissa). The dotted line is a guide for the eye.

apply, the chemical potential satisfies $\exp (\mu \beta) \ll 1$. To ascertain the strength of the deviations from fully classical behavior $\mu$ is determined from the implicit equation

$$
\rho=\frac{g M^{3 / 2}}{\sqrt{2} \pi^{2} \hbar^{3}} \int_{0}^{\infty} \frac{d \varepsilon \sqrt{\varepsilon}}{\exp \beta(\varepsilon-\mu)-1},
$$

where $g=2 I+1$ stands for the total spin. Solution of Eq. (34) for the two extreme densities and the corresponding temperature yields $\mu=-0.988 \mathrm{meV}$ and $\mu=-0.900 \mathrm{meV}$. This yields values of $\exp (\mu \beta)$ of 0.5 and 0.53 , respectively, that clearly show that the condition for the validity of Boltzmann statistics does not hold and are comparable to figures corresponding to normal liquid ${ }^{4} \mathrm{He}$ at $2.5 \mathrm{~K}$ and a density of $0.0218 \AA^{-3}$. From Eqs. (32) and (33) one can estimate how much of the increase in kinetic energy is due to a variation of the chemical potential with increasing density. The results show that the observed rise in kinetic energy with increasing density cannot be ascribed to an effect driven by the variation of the chemical potential only.

The large increase in kinetic energy can be also used to explain the rise in phonon frequency versus pressure recently found on the basis of path-integral centroid molecular dynamics simulations. ${ }^{13}$ There, it was found that the slope of the $\omega_{Q}$ phonon frequencies for wave vectors below $\approx 0.7 \AA^{-1}$, where the dispersion relation is almost linear, increases by some $11.6 \%$ while the increase due to density is obviously far more modest. From data on classical liquids such as molten metals ${ }^{26}$ we know that such phonon frequencies are generally close to those followed by the fourth moment of the response function

$$
\omega_{l}^{2}=\frac{3 \hbar^{2} Q^{2}}{M \beta}+\Omega(0)^{2}+\Omega(Q)^{2}
$$

where $\Omega(Q)$ and $\Omega(0)$ are quantities obtainable from the interaction potential and pair distribution function. On physical grounds, the meaning of such frequency is best understood from

$$
\omega_{l}^{2}=\frac{\hbar^{2} Q^{2} C_{11}(Q)}{M \rho},
$$

where $C_{11}(Q)$ plays the role of a generalized longitudinal elastic constant. From here we see that the expected change in frequency due to density should scale as the square root of the mass density and therefore should not largely exceed $4 \%$.

An expression adequate to describe an equivalent quantity for a quantum fluid is given by

$$
\omega_{l}^{2}=\left(\frac{\hbar^{2} Q^{2}}{2 M}\right)^{2}+\left(\frac{2 \hbar^{2} Q^{2}}{M}\right)\left\langle K_{E}\right\rangle+\Omega(0)^{2}+\Omega(Q)^{2},
$$

where the additional term on $\left\langle K_{E}\right\rangle$ may serve to explain such an anomalous increase.

\section{B. Test of the CDM prediction}

Our experimental data on the momentum distribution $n(k)$ of liquid para-hydrogen permit a pertinent comparison to theoretical results derived from the structural decomposition (3). At present, numerical results of theoretical calculations within CDM theory ${ }^{14}$ are available for the factor $n_{\mathrm{c}}$ and the unit-normalized phase-phase correlation function $Q(r) / Q(0)$ at temperature $T=16 \mathrm{~K}$ and particle-number density $\rho$ $=0.021 \AA^{-3}$. These parameters differ only slightly from the experimental data $16.5 \mathrm{~K}$ and $0.02235 \AA^{-3}$ (corresponding to a pressure of 1 bar), respectively. The difference affects somewhat the size of the total kinetic energy, which increases with increasing density (about 5-8 $\mathrm{K}$ from density $0.021 \AA^{-3}$ to $0.02235 \AA^{-3}$ ). Under the thermal conditions adopted, the thermal wavelength $\lambda$ appearing in Eq. (3) is $\lambda=3.09 \AA$. The theoretical phase-phase correlation function is very well approximated by the Gaussian $Q(r) / Q(0)$ $=\exp \left[-\pi\left(r / \lambda_{p}\right)^{2}\right]$ with an effective wavelength $\lambda_{p}=3.73 \AA$. The corresponding total kinetic energy has the theoretical value $58.6 \mathrm{~K}$, which leads to a strength factor $n_{\mathrm{c}} \simeq 0.118$. The difference between the theoretical value for the kinetic energy per particle and the experimental data is about $10 \mathrm{~K}$ as expected due to the density difference. However, the various correlation functions and distributions at a given relative distance $r$ or wave number $k$ are much less affected. The upper frame of Fig. 11 displays the experimental results of fit (29) on the energy distribution $k^{2} n(k)$, in arbitrary units. They are compared with the corresponding classical Maxwell curve $k^{2} n_{\mathrm{G}}(r)$ given by expression (29) without the bracket factor. We find that the experimental energy distribution has its peak value 0.191 at $k \simeq 1.72 \AA^{-1}$ and is shifted to smaller wave numbers compared with the Maxwell-Gauss distribution. The theoretical results of these energy distributions show the same behavior. Due to the lower density adopted in the theoretical calculations, the peak value of the distribution $k^{2} n(k)$ is about 0.18 and located at $k \simeq 1.6 \AA^{-1}$. A detailed comparison of the experimental data with the theoretical results on the energy distribution $k^{2} n(k)$ is displayed in the lower frame of Fig. 11. Shown are the theoretical and experimental results of the difference (5). They agree fairly 


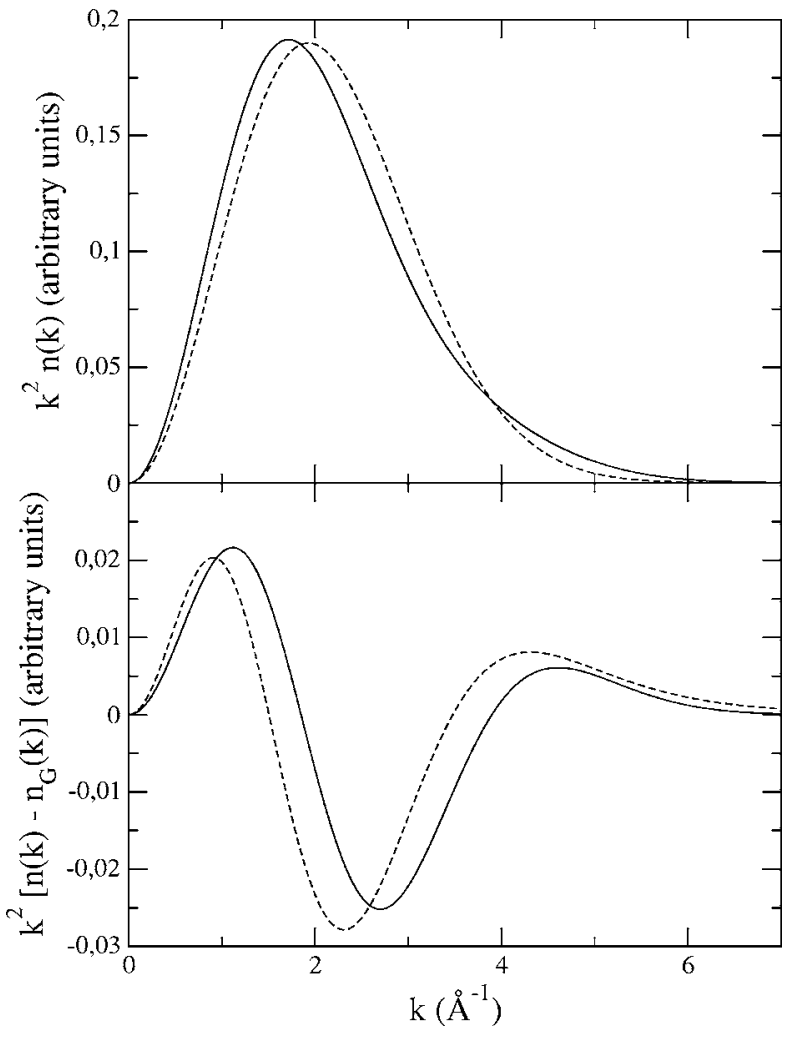

FIG. 11. Upper frame: experimental results of the kinetic energy distribution $k^{2} n(k)$ (in arbitrary units) at $T=16.5 \mathrm{~K}$ and pressure 1 bar (particle-number density $0.02235 \AA^{-3}$ ). They are compared with the corresponding Maxwell-Gauss results $k^{2} n_{\mathrm{G}}(k)$ based on the fit (29) without the bracket factor (dashed curve); for a comparison with theoretical results see the text. Lower frame: comparison of experimental and theoretical results for the difference (5) that measures the deviation of the kinetic energy distribution $k^{2} n(k)$ of liquid para-hydrogen from the classical Maxwell-Gauss distribution $k^{2} n_{\mathrm{G}}(k)$ associated with the same total kinetic energy, $\left\langle E_{K}^{G}\right\rangle_{T}$ $=\left\langle E_{K}\right\rangle_{T}$. The deformation is due to the indistinguishability of the $\mathrm{H}_{2}$ molecules (solid line, experimental results at $1 \mathrm{bar}$; dashed line, theoretical results).

well. Both curves tell that quantum mechanics leads to an increased energy distribution at wave numbers $k<1.5 \AA^{-1}$ compared to a classical Maxwell behavior due to an increased number of molecules in this region.

\section{Pressure dependence of the strength of FSE interactions}

The density dependence of the parameters governing the strength of the FSE interactions-namely, $\bar{a}_{3}$ - can be qualitatively understood from the known moment sum rules of the $R(Q, t)$ broadening function. The first nontrivial moment, as expressed in Eq. (18), becomes ${ }^{27}$

$$
\mu_{3}(Q)=\frac{\hbar^{4} Q^{2}}{2 M^{2}} \rho \int d \mathbf{r} g(r)(\hat{\mathbf{Q}} \cdot \nabla)^{2} \nu(r),
$$

where $\rho$ stands for the particle density and $g(r)$ is the radial distribution. In other words, the third moment of the FSE broadening function is given by a function written in terms of the radial distribution and interatomic potential, two quan- tities not included in the impulse approximation. Since the potential depends only on $r$, Eq. (38) becomes

$$
\mu_{3}(Q)=\frac{2 \pi}{3} \frac{\hbar^{4} Q^{2}}{M^{2}} \rho \int d r r^{2} g(r) \frac{\partial^{2} \nu(r)}{\partial r^{2}},
$$

so comparing with Eq. (18),

$$
\bar{a}_{3}(Q)=\frac{2 \pi \hbar^{2}}{3} \rho \int d r r^{2} g(r) \frac{\partial^{2} \nu(r)}{\partial r^{2}} .
$$

The density dependence of $g(r)$ is known from our previous work, ${ }^{13}$ and the potential can be obtained from various sources. ${ }^{28-30}$ In Fig. 7 the fitted parameter $\bar{a}_{3}$ is compared with Eq. (40). The calculation is especially sensitive to the repulsive part of the potential. In the calculation we employed the same parametrization as in Refs. 29 and 30 with an increment of $0.6 \%$ in the parameter $b$ entering the repulsive part of the potential. In doing so we account for the general trend of increasing the FSE with rising density, which is expected due to the shift of the spectral distribution to higher frequencies with increasing density.

\section{CONCLUSION}

The results here reported on depict a behavior for the kinetic energy that increases with density by an amount difficult to account for using standard statistical mechanics tools. Our data serve to explain in part the rather different estimates for this quantity reported for liquid and solid hydrogen at $10 \mathrm{~K}$ and $17 \mathrm{~K},{ }^{3}$ where $\left\langle E_{K}\right\rangle$ is found to increase from $(63 \pm 6) \mathrm{K}$ in the liquid up to $(76 \pm 9) \mathrm{K}$ for the crystal. Such an increase of about $20 \%$ mostly comes from the density increase of about $15 \%$ brought forward by crystallization.

The most evident signatures of nonclassical effects on $n(k)$ such as the non-Gaussian correction get substantially diminished with increasing density. In other words, the net effect of the increasing role of interparticle interactions when the density increases is to move particles occupying lowmomentum states towards others with higher momenta. The phenomenon is then understood on the same grounds than that leading to a redistribution of the momentum states due to molecular interactions as described using Eqs. (2) and (3).

Our results also bear some resemblance to those recently reported on for overpressurized liquid helium. ${ }^{31}$ In fact, such results report on an increase in energy per particle with increasing density that follows a polynomial law with terms up to fourth order. In contrast, the most remarkable quantum feature such as the Bose condensate fraction decays exponentially with increasing density. In fact, superfluidity disappears beyond pressures of 40 bars. ${ }^{32,33}$ This behavior is also followed by that found for the static structure factor $S(Q)$. Its main peak located at about $2 \AA^{-1}$ sharpens and significantly increases in height while its low- $Q$ limiting behavior decreases with increasing density following the limit $\hbar Q / 2 m c$ that is governed by the increase in sound velocity $c$. In turn, the behavior of $S(Q)$ translates into a decrease and a shift towards higher-momentum transfers of the roton peak, due to the second-moment sum rule. 
*Electronic address: javier@cab.cnea.gov.ar

${ }^{\dagger}$ Also at Consejo Superior de Investigaciones Científicas, Serrano 123 E-28006 Madrid, Spain.

${ }^{1}$ H. R. Glyde and W. G. Stirling, in Momentum Distributions, edited by R. N. Silver and P. E. Sokol (Plenum, New York, 1989), p. 123; H. R. Glyde and E. C. Svensson, in Neutron Scattering, edited by D. L. Price and K. Sköld, Methods of Experimental Physics, Vol. 23 (Academic Press, New York, 1987), Pt. B, p. 303.

${ }^{2}$ T. R. Sosnick, W. M. Snow, and P. E. Sokol, Phys. Rev. B 41, 11185 (1990); V. F. Sears, Phys. Rev. 185, 200 (1969).

${ }^{3}$ W. Langel, D. L. Price, R. O. Simmons, and P. E. Sokol, Phys. Rev. B 38, 11275 (1988).

${ }^{4}$ C. Andreani, D. Colognesi, and E. Pace, Phys. Rev. B 60, 10008 (1999); C. Andreani, Physica B 350, 231 (2004).

${ }^{5}$ F. J. Mompean, M. Garcia-Hernandez, F. J. Bermejo, and S. M. Bennington, Phys. Rev. B 54, 970 (1996).

${ }^{6}$ J. Mayers, Phys. Rev. Lett. 71, 1553 (1993).

${ }^{7}$ M. Zoppi, D. Colognesi, and M. Celli, Eur. Phys. J. B 23, 171 (2001); U. Bafile, M. Zoppi, M. Celli, R. Magli, A. C. Evans, and J. Mayers, Physica B 217, 50 (1996).

${ }^{8}$ M. Mukherjee, F. J. Bermejo, S. M. Bennington, and B. Fåk, Phys. Rev. B 57, R11031 (1998).

${ }^{9}$ F. J. Bermejo, K. Kinugawa, C. Cabrillo, S. M. Bennington, B. Fåk, M. T. Fernández-Díaz, P. Verkerk, J. Dawidowski, and R. Fernández-Perea, Phys. Rev. Lett. 84, 5359 (2000).

${ }^{10}$ F. J. Bermejo, B. Fåk, S. M. Bennington, R. Fernández-Perea, C. Cabrillo, J. Dawidowski, M. T. Fernández-Díaz, and P. Verkerk, Phys. Rev. B 60, 15154 (1999).

${ }^{11}$ M. Mukherjee, F. J. Bermejo, B. Fåk, and S. M. Bennington, Europhys. Lett. 40, 153 (1997).

${ }^{12}$ I. F. Silvera, Rev. Mod. Phys. 52, 393 (1980); J.-L. Li, G.-M. Rignanese, E. K. Chang, X. Blase, and S. G. Louie, Phys. Rev. B 66, 035102 (2002); E. Gregoryanz, A. F. Goncharov, K. Matsuishi, H.-K. Mao, and R. J. Hemley, Phys. Rev. Lett. 90, 175701 (2003); N. A. Tahir et al., Phys. Rev. B 67, 184101 (2003); R. Redmer, G. Röpke, S. Kuhlbrodt, and H. Reinholz, ibid. 63, 233104 (2001); W. J. Nellis, S. T. Weir, and A. C. Mitchell, ibid. 59, 3434 (1999); M. Ross, ibid. 54, R9589 (1996); S. T. Weir, A. C. Mitchell, and W. J. Nellis, Phys. Rev. Lett. 76, 1860 (1996); I. I. Mazin and R. E. Cohen, Phys. Rev. B 52, R8597 (1995).

${ }^{13}$ F. J. Bermejo, K. Kinugawa, J. Dawidowski, and C. Cabrillo, Chem. Phys. 317, 198 (2005).
${ }^{14}$ R. Pantfoerder, T. Lindenau, and M. L. Ristig, J. Low Temp. Phys. 108, 245 (1997); T. Lindenau, M. L. Ristig, K. A. Gernoth, J. Dawidowski, and F. J. Bermejo, in Advances in Quantum Many-Body Theory, edited by E. Susana Hernandez and Horacio M. Cataldo (World Scientific, Singapore, in press).

${ }^{15}$ E. Feenberg, Theory of Quantum Fluids (Academic Press, New York, 1969); M. L. Ristig, in From Nuclei to Particles, Proceedings of the International School of Physics, Enrico Fermi, Course LVII, Varenna 1981, edited by A. Molinari (NorthHolland, Amsterdam, 1982).

${ }^{16}$ J. Dawidowski, F. J. Bermejo, and J. R. Granada, Phys. Rev. B 58, 706 (1998).

${ }^{17}$ V. F. Sears, Adv. Phys. 24, 1 (1975).

${ }^{18}$ J. R. D. Copley, Comput. Phys. Commun. 7, 289 (1974); J. R. D. Copley, P. Verkerk, A. A. Van Well, and H. Fredrikze, ibid. 40, 337 (1986).

${ }^{19}$ W. D. Seiffert (unpublished).

${ }^{20}$ J. R. Granada, V. H. Gillette, M. E. Pepe, and M. M. Sbaffoni, J. Neutron Res., 11, 25 (2003).

${ }^{21}$ J. Dawidowski, J. R. Santisteban, and J. R. Granada, Physica B 271, 212 (1999).

${ }^{22}$ H. R. Glyde, Phys. Rev. B 50, 6726 (1994); S. O. Diallo, J. V. Pearce, R. T. Azuah, and H. R. Glyde, Phys. Rev. Lett. 93, 075301 (2004).

${ }^{23}$ J. Dawidowski, F. J. Bermejo, M. L. Ristig, B. Fåk, C. Cabrillo, R. Fernández-Perea, K. Kinugawa, and J. Campo, Phys. Rev. B 69, 014207 (2004).

${ }^{24}$ V. F. Sears, Can. J. Phys. 44, 1279 (1966).

${ }^{25}$ J. A. Young and J. U. Koppel, Phys. Rev. 135, A603 (1964).

${ }^{26}$ C. Cabrillo, F. J. Bermejo, A. Maira-Vidal, R. Fernandez-Perea, S. M. Bennington, and D. Martin, J. Phys.: Condens. Matter 16, 309 (2004).

${ }^{27}$ F. Mazzanti, J. Boronat, and A. Polls, Phys. Rev. B 53, 5661 (1996).

${ }^{28}$ I. F. Silvera and V. V. Goldman, J. Chem. Phys. 69, 4209 (1978).

${ }^{29}$ M. Boninsegni, New J. Phys. 7, 78 (2005).

${ }^{30}$ F. Operetto and F. Pederiva, Phys. Rev. B 69, 024203 (2004).

${ }^{31}$ L. Vranjes, J. Boronat, J. Casulleras, and C. Cazorla, Phys. Rev. Lett. 95, 145302 (2005).

${ }^{32}$ K. Yamamoto, H. Nakashima, Y. Shibayama, and K. Shirahama, Phys. Rev. Lett. 93, 075302 (2004).

${ }^{33}$ J. V. Pearce, J. Bossy, H. Schober, H. R. Glyde, D. R. Daughton, and N. Mulders, Phys. Rev. Lett. 93, 145303 (2004). 